PROCEEDINGS OF THE

AMERICAN MATHEMATICAL SOCIETY

Volume 137, Number 11, November 2009, Pages 3669-3678

S 0002-9939(09)09331-9

Article electronically published on June 16, 2009

\title{
TOEPLITZ AND HANKEL OPERATORS AND DIXMIER TRACES ON THE UNIT BALL OF $\mathbb{C}^{n}$
}

\author{
MIROSLAV ENGLIŠ, KUNYU GUO, AND GENKAI ZHANG
}

(Communicated by Marius Junge)

\begin{abstract}
We compute the Dixmier trace of pseudo-Toeplitz operators on the Fock space. As an application we find a formula for the Dixmier trace of the product of commutators of Toeplitz operators on the Hardy and weighted Bergman spaces on the unit ball of $\mathbb{C}^{d}$. This generalizes an earlier work of Helton-Howe for the usual trace of the anti-symmetrization of Toeplitz operators.
\end{abstract}

\section{INTRODUCTION}

In the present paper we will study the Dixmier trace of a class of Toeplitz and Hankel operators on the Hardy and weighted Bergman spaces on the unit ball of $\mathbb{C}^{d}$. We give a brief account of our problem and explain some motivations. Consider the Bergman space $L_{a}^{2}(D)$ of holomorphic functions on the unit disk $D$ in the complex plane. For a bounded function $f$ let $T_{f}$ be the Toeplitz operator on $L_{a}^{2}(D)$. It is well-known that for a holomorphic function $f$ in a neighborhood of $D$ the commutator $\left[T_{f}^{*}, T_{f}\right]$ is of trace class and the trace is given by the square of the Dirichlet norm of $f$,

$$
\operatorname{tr}\left[T_{f}^{*}, T_{f}\right]=\int_{D}\left|f^{\prime}(z)\right|^{2} d m(z)
$$

which is one of the best known Möbius invariant integrals. This formula actually holds for Toeplitz operators on any Bergman space on a bounded domain with the area measure replaced by any reasonable measure 2 . There is a significant difference between Toeplitz operators on the unit disk and on the unit ball $B=B^{d}$ in $\mathbb{C}^{d}, d>1$. Let $\mathcal{L}^{p}$ be the Schatten - von Neumann class of $p$-summable operators. The commutator $\left[T_{f}^{*}, T_{f}\right]$ on the weighted Bergman space, say for holomorphic functions $f$ in a neighborhood of the closed unit disk, is in the Schatten - von Neumann class $\mathcal{L}^{p}$, for $p>\frac{1}{2}$, and is zero if it is in $\mathcal{L}^{p}$, for $p \leq \frac{1}{2}, \frac{1}{2}$ being called the cut-off; on the Hardy space $\left[T_{f}^{*}, T_{f}\right]$ can be in any Schatten - von Neumann class

Received by the editors February 19, 2007, and, in revised form, July 12, 2007.

2000 Mathematics Subject Classification. Primary 32A36; Secondary 47B35, 47B06.

Key words and phrases. Schatten - von Neumann classes, Macaev classes, trace, Dixmier trace, Toeplitz operators, Hankel operators, pseudo-Toeplitz operators, pseudo-differential operators, boundary CR operators, invariant Banach spaces.

The research of the first author was supported by GA ČR grant No. 201/06/128 and AV ČR Institutional Research Plan No. AV0Z10190503, of the second author by NSFC(10525106) and NKBRPC(2006CB805905), and of the third author by the Swedish Science Council (VR) and SIDA-Swedish Research Links.

(C)2009 American Mathematical Society Reverts to public domain 28 years from publication 
$\mathcal{L}^{p}$, for $p>0$. See 14] and [16] for the case of a Hardy space and 1] for the case of a weighted Bergman space. However for $d>1$, it is in $\mathcal{L}^{p}$ for $p>d$, with $p=d$ being the cut-off, both on the weighted Bergman spaces and on the Hardy space. Thus no trace formula was expected for the commutators. Nevertheless Helton and Howe [9] were able to find an analogue of the previous formula. They showed, for smooth functions $f_{1}, \cdots, f_{2 d}$ on the closed unit ball, that the anti-symmetrization $\left[T_{f_{1}}, T_{f_{2}}, \cdots, T_{f_{2 d}}\right]$ of the $2 d$ operators $T_{f_{1}}, T_{f_{2}}, \cdots, T_{f_{2 d}}$ is of trace class and found that

$$
\operatorname{tr}\left[T_{f_{1}}, T_{f_{2}}, \cdots, T_{f_{2 d}}\right]=\int_{B} d f_{1} \wedge d f_{2} \cdots \wedge d f_{2 d} .
$$

On the other hand, we observe that $\left[T_{f}, T_{g}\right]$ is, for smooth functions $f$ and $g$, in the Macaev class $\mathcal{L}^{d, \infty}$ (which is an analogue of the Lorentz space $L^{d, \infty}$ ); thus the product of $d$ such commutators $\left[T_{f_{1}}, T_{g_{1}}\right]\left[T_{f_{2}}, T_{g_{2}}\right] \cdots\left[T_{f_{d}}, T_{g_{d}}\right]$ is in $\mathcal{L}^{1, \infty}$ and hence has a Dixmier trace. One of the goals of the present paper is to prove the following formula for the Dixmier trace of this product of commutators:

$$
\operatorname{tr}_{\omega}\left[T_{f_{1}}, T_{g_{1}}\right] \cdots\left[T_{f_{d}}, T_{g_{d}}\right]=\frac{1}{d !} \int_{S}\left\{f_{1}, g_{1}\right\} \cdots\left\{f_{d}, g_{d}\right\} .
$$

Here $\{f, g\}$ is the Poisson bracket of $f$ and $g$; its restriction to the boundary $S$ of $B$ depends only on the boundary values of $f$ and $g$ and can be expressed in terms of the boundary $C R$ operators. This can be viewed as a generalization of the Helton-Howe theorem. We apply our result also to Hankel operators and obtain a formula for the Dixmier trace of the $d$-th power of the square modulus of the Hankel operators $H_{f}^{*} H_{\bar{f}}$ for holomorphic functions $f$. Namely we have

$$
\operatorname{tr}_{\omega}\left|H_{\bar{f}}\right|^{2 d}=\operatorname{tr}_{\omega}\left(\left[T_{\bar{f}}, T_{f}\right]^{d}\right)=\frac{1}{d !} \int_{S}\left(|\nabla f|^{2}-|R f|^{2}\right)^{d} .
$$

This provides a boundary $\mathcal{L}^{d, \infty}$ result for the Schatten - von Neumann $\mathcal{L}^{p}(p>d)$ properties of the square modulus of the Hankel operators (see [3, [5] and [19]). We mention also, besides the above results on exact norms, that there are exact formulas proved by Janson, Upmeier and Wallstén [12] on the Schatten - von Neumann $\mathcal{L}^{p}$ norm of the Hankel operators on the unit circle for $p=2,4,6$, and by Peetre [13] on $\mathcal{L}^{4}$-norms of Hankel forms on Fock spaces [11.

There has been an intensive study of Dixmier trace and residue trace of pseudodifferential operators, mostly on compact manifolds where the analysis is relatively easier; see e.g. [4 and [15] and the references therein. Thus the Toeplitz operators on Hardy spaces on the boundary of a bounded strictly pseudo-convex domain can be treated using the techniques developed there. The Hankel and Toeplitz operators on Bergman spaces, generally speaking, behave rather differently from those on a Hardy space, and the result of Howe [10 roughly speaking proves that Toeplitz operators of certain classes can be treated similarly to those in the Hardy space case (also called the de Monvel - Howe compactification [6]). Our result can thus be viewed as a generalization of the compactification to weighted Bergman spaces and an application of the ideas in [7] of computing Dixmier traces. In particular our Theorem 4.1 is closely related to the results in [4, where the residue trace of pseudo-differential operators of a certain class is computed; here we use the Weyl transforms and they differ from pseudo-differential operators of lower order, so that Theorem 4.1 can also be obtained from 4 . provided one proves that the lower order terms are of trace class. 
In another paper we will study the Dixmier trace for Toeplitz operators on a general strongly pseudo-convex domain. One of the authors, G. Zhang, would also like to thank Professor Richard Rochberg and Professor Harald Upmeier for introducing him to the work of Connes [8, Chapter IV.2] on Dixmier traces of pseudo-differential operators.

\section{TOEPLitZ OPERATORS ON BERgMAN SPACES AND THEIR REALIZATION AS PSEUDO-TOEPLITZ OPERATORS ON FOCK SPACES}

Let $d m(z)$ be the Lebesgue measure on $\mathbb{C}^{d}$ and consider the weighted measure

$$
d \mu_{\nu}=C_{\nu}\left(1-|z|^{2}\right)^{\nu-d-1} d m(z),
$$

where $C_{\nu}$ is the normalizing constant to make $d \mu_{\nu}$ a probability measure and $\nu>d$. We let $\mathcal{H}_{\nu}$ be the corresponding Bergman space of holomorphic functions on $B$. We will also consider the Hardy space of square integrable functions on $S$ which are holomorphic on $B$. This can be viewed as the analytic continuation of $\mathcal{H}_{\nu}$ at $\nu=d$. Thus we assume throughout this paper that $\nu \geq d$.

Let $f$ be a bounded smooth function on $\bar{B}$, the closure of $B$. The Toeplitz operator $T_{f}$ on $\mathcal{H}_{\nu}$ with symbol $f$ is defined by

$$
T_{f} g=P(f g),
$$

where $P$ is the Bergman or the Hardy projection for $\nu>d$ and $\nu=d$, respectively.

As was shown by Howe [10] there is a more flexible and effective way of studying the spectral properties of Toeplitz operators with smooth symbol, by using the theories of representations of the Heisenberg group and of pseudo-differential operators. We will adopt that approach. We will be very brief and refer to [10] and [18, Chapter XII] for details. So let $H_{n}=\mathbb{C}^{d} \times T$ be the Heisenberg group as in loc. cit. The Heisenberg group has an irreducible representation, $\rho$, on the Fock space $\mathcal{F}$ consisting of entire functions $f$ on $\mathbb{C}^{d}$ such that

$$
\int_{\mathbb{C}^{d}}|f(z)|^{2} e^{-\pi|z|^{2}} d m(z)<\infty .
$$

The action of the Heisenberg group is explicitly given as follows. For $w \in \mathbb{C}^{d}$ viewed as an element in $H_{d}$,

$$
\rho(w) f\left(w^{\prime}\right)=e^{-\pi / 2|w|^{2}+\pi w^{\prime} \cdot \bar{w}} f\left(w^{\prime}-w\right),
$$

where $w^{\prime} \cdot \bar{w}$ is the Hermitian inner product on $\mathbb{C}^{d}$. The action of $T$ is by rotation.

Identifying the Lie algebra $\mathfrak{h}$ of the Heisenberg group with $\mathbb{R}^{2 n} \oplus \mathbb{R}$ and thus $\mathbb{R}^{2 n}$ with a subspace of the Lie algebra, we get an action of $\mathbb{R}^{2 n}$ as holomorphic differential operators on $\mathcal{F}$, which extends from $\mathfrak{h}$ to the whole enveloping algebra $\mathfrak{U}(\mathfrak{h})$ and which will also be denoted by $\rho$. In particular, taking the basis elements $\partial_{j}=\partial / \partial w_{j}$ and $\bar{\partial}_{j}=\partial / \partial \bar{w}_{j}$ of $\mathbb{R}^{2 n}$ we have

$$
\rho\left(\partial_{j}\right) f(w)=-\partial_{j} f(w), \quad \rho\left(\bar{\partial}_{j}\right) f(w)=\pi w_{j} f(w) .
$$

Following the notation in [10, let $\Delta \in \mathfrak{U}(\mathfrak{h})$ be the element

$$
\Delta=\frac{1}{2}\left(\partial_{j} \cdot \bar{\partial}_{j}+\bar{\partial}_{j} \cdot \partial_{j}\right)
$$


Then $\rho(\Delta)$ acts on $\mathcal{F}$ as a diagonal self-adjoint operator [10], under the orthogonal basis $\left\{w^{\alpha}, \alpha=\left(\alpha_{1}, \cdots, \alpha_{d}\right)\right\}$, viz.

$$
\rho(\Delta) w^{\alpha}=-\pi\left(|\alpha|+\frac{d}{2}\right) w^{\alpha}
$$

Let $F(z)$ be a function on $\mathbb{C}^{d}$ (viewed as a function on the Heisenberg group). The Weyl transform $\rho(F)$ of $F$ is defined by

$$
\rho(F)=\int_{\mathbb{C}^{d}} F(w) \rho(w) d m(w) .
$$

To understand the operator theoretic properties of $\rho(F)$ we will need the Fourier transform of $F$. Let $\hat{F}$ be the (symplectic-) Fourier transform of $F$,

$$
\hat{F}\left(w^{\prime}\right)=2^{-d} \int_{\mathbb{C}^{d}} F(w) e^{\pi i \operatorname{Im} w^{\prime} \cdot \bar{w}} d m(w),
$$

and let $F * G$ be the symplectic convolution

$$
F * G(w)=\int_{\mathbb{C}^{d}} F(z) G(w-z) e^{\pi i \operatorname{Im} w \cdot \bar{z}} d m(z) .
$$

We recall that

$$
\widehat{F * G}=F * \hat{G}
$$

and

$$
\rho(F) \rho(G)=\rho(F * G)
$$

for an appropriate class of functions. A well-known theorem of Calderón-Vaillancourt states that if $\hat{F}$ and all its derivatives are bounded, then $\rho(F)$ can be defined as a bounded operator on $\mathcal{F}$.

We will need a finer class of symbols introduced by Howe, corresponding to the so-called pseudo-Toeplitz operators. Let

$$
\mathcal{P} \mathcal{T}(m, \mu)=\left\{F \in \mathcal{S}^{*}\left(\mathbb{C}^{d}\right):\left|\partial^{\alpha} \bar{\partial}^{\beta} \hat{F}\right| \leq C_{\alpha \beta}(1+|w|)^{m-\mu(|\alpha|+|\beta|)}\right\}
$$

and

$$
\begin{aligned}
& \mathcal{P} \mathcal{T}_{\text {rad }}(m, \mu)=\{F \in \mathcal{P} \mathcal{T}(m, \mu): \\
&\left.\hat{F}=(1-g(|w|)) \psi\left(\frac{w}{|w|}\right)|w|^{m}+D_{1}, D_{1} \in \mathcal{P} \mathcal{T}(m-\mu, \mu)\right\} .
\end{aligned}
$$

Here $g$ is a smooth function on $\mathbb{R}$ such that $0 \leq g(t) \leq 1$ on $\mathbb{R}, g(t)=0$ for $|t| \geq 2$ and $g(t)=1$ for $0 \leq t \leq 1$.

For $F \in \mathcal{P} \mathcal{T}_{\text {rad }}(m, \mu)$ we will call

$$
\sigma_{m}(F):=\psi\left(\frac{w}{|w|}\right)|w|^{m}
$$

its principal symbol. It can be obtained, up to the factor $|w|^{m}$, by

$$
\psi(w)=\lim _{t \rightarrow \infty} t^{-m} \hat{F}(t w), \quad w \in S .
$$

Following Howe [10, we will call $\rho(F), F \in \mathcal{P} \mathcal{T}(m, \mu)$, a pseudo-Toeplitz operator of order $m$ and smoothness $\mu$. One has [10, Lemma 4.2.2]

$$
F \in \mathcal{P} \mathcal{T}\left(m_{1}, \mu\right), G \in \mathcal{P} \mathcal{T}\left(m_{2}, \mu\right) \Longrightarrow F * G \in \mathcal{P} \mathcal{T}\left(m_{1}+m_{2}, \mu\right) .
$$


We will realize the Toeplitz operators $T_{f}$ on $\mathcal{H}_{\nu}$ for $f$ on $B$ (or on $S$ for the Hardy space) as Weyl transforms $\rho(F)$ of certain symbols $F$ on $\mathbb{C}^{d}$. First we notice that

$$
e_{\beta}:=\left(\frac{(\nu)_{|\beta|}}{\beta !}\right)^{\frac{1}{2}} z^{\beta}
$$

form an orthonormal basis of $\mathcal{H}_{\nu}$, and so do

$$
E_{\beta}:=\left(\frac{1}{\pi^{|\beta|} \beta !}\right)^{\frac{1}{2}} w^{\beta}
$$

for $\mathcal{F}$. (Here $(\nu)_{j}:=\nu(\nu+1) \ldots(\nu+j-1)$ is the usual Pochhammer symbol.) Thus the map

$$
U: e_{\beta} \rightarrow E_{\beta}
$$

is a unitary operator. First we will find the action of the elementary Toeplitz operators $T_{z^{\alpha}}$ under the intertwining map $U$.

Lemma 2.1. The operator $U T_{z^{\alpha}} U^{*}$ on $\mathcal{F}$ is given by

$$
U T_{z^{\alpha}} U^{*}=\rho(z)^{\alpha} \rho\left(\pi^{|\alpha|}\left(\nu-\frac{d}{2}-\frac{1}{\pi} \Delta\right)_{|\alpha|}\right)^{-1 / 2} .
$$

This can be proved by direct computation. Indeed we have

$$
T_{z^{\alpha}} e_{\beta}=\left(\frac{(\beta)_{\alpha}}{(\nu+|\beta|)_{|\alpha|}}\right)^{\frac{1}{2}} e_{\beta+\alpha}
$$

and the right hand side (2.6) can be easily computed by (2.1) and (2.2).

By using the previous lemma we have then the following result, which was proved by Howe [10, Proposition 4.2.3] in the case when $\nu=d+1$; the general case of $\nu \geq d$ is essentially the same.

Proposition 2.2. Let $f \in C^{\infty}(S)$ and let $\tilde{f}$ be a $C^{\infty}$ extension to $B$ and $T_{\tilde{f}}$ the Toeplitz operator on $\mathcal{H}_{\nu}$. Then under the unitary equivalence of $\mathcal{H}_{\nu}$ and the Fock space $\mathcal{F}$ on $\mathbb{C}^{d}$, the Toeplitz operators are pseudo-Toeplitz operators with radial asymptotic limits $\mathcal{P} \mathcal{T}_{\text {rad }}(0,1)$. More precisely, there exists $F \in \mathcal{P} \mathcal{T}_{\text {rad }}(0,1)$ such that $U T_{\tilde{f}} U^{*}=\rho(F)$, and $f(\zeta)=\lim _{t \rightarrow \infty} \hat{F}(t \zeta)$ for each $\zeta \in S$.

\section{Schatten - von Neumann properties of pseudo-Toeplitz operators}

Recall that the Schatten - von Neumann class $\mathcal{L}^{p}, p \geq 1$, consists of compact operators $T$ such that the eigenvalues $\left\{\mu_{n}\right\}$ of $|T|=\left(T^{*} T\right)^{\frac{1}{2}}$ are $p$-summable, $\sum \mu_{n}^{p}<\infty$. In particular $\mathcal{L}^{2}$ is the Hilbert-Schmidt class, $\mathcal{L}^{1}$ the trace class and $\mathcal{L}^{\infty}$ are the compact operators. For $1<p<\infty, 1 \leq q \leq \infty$, the Macaev class $\mathcal{L}^{p, q}$ is obtained by the real interpolation between $\mathcal{L}^{1}$ and $\mathcal{L}^{\infty}$. However, we will need the Macaev class $\mathcal{L}^{1, \infty}$, which consists 11 of all compact operators such that, if $\mu_{1} \geq \mu_{2} \geq \ldots$,

$$
\sum_{n=1}^{N} \mu_{n}=O(\log N)
$$

There exists a linear functional on the space $\mathcal{L}^{1, \infty}$ that resembles the usual trace, called the Dixmier trace. Its definition is rather involved and we refer to 8 ,

\footnotetext{
${ }^{1}$ Sometimes this ideal is denoted by $\mathcal{L}_{\Omega}$, the notation $\mathcal{L}^{1, \infty}$ being reserved for the (smaller) class of operators for which $\mu_{n}=O(1 / n)$. Our notation follows Connes' book 8 .
} 
Chapter IV] for details. Let $C_{b}\left(\mathbb{R}_{+}\right)$be the space of bounded continuous functions on $\mathbb{R}_{+}$and $C_{0}\left(\mathbb{R}_{+}\right)$the subspace of functions vanishing at $\infty$. Let $\omega$ be a positive linear functional on the quotient space $C_{b}\left(\mathbb{R}_{+}\right) / C_{0}\left(\mathbb{R}_{+}\right)$such that $\omega(1)=1$. For a positive compact operator $T \in \mathcal{L}^{1, \infty}$ with eigenvalues $\left\{\mu_{n}\right\}$, extend $\mu_{n}$ to a step function on $\mathbb{R}_{+}$and let $M_{T}(\lambda)$ be its Cesáro mean, which is a bounded continuous function on $\mathbb{R}^{+}$. The Dixmier trace of $T$ is then defined by

$$
\operatorname{tr}_{\omega} T=\omega\left(M_{T}\right) \text {. }
$$

It is then extended to all of $\mathcal{L}^{1, \infty}$ by linearity. In particular it is bounded and vanishes on trace class operators. The fact that we will need is that

$$
\operatorname{tr}_{\omega} T=\lim _{N \rightarrow \infty} \frac{1}{\log N} \sum_{n=1}^{N} \mu_{n}(T)
$$

if $T$ is a positive operator and if the right hand side exists.

Lemma 3.1. For any $c \geq 0$ the operator $(c-\rho(\Delta))^{-d}=\rho\left(c \delta_{0}-\Delta\right)^{-d}$ is in the Macaev class $\mathcal{L}^{1, \infty}$.

Proof. It follows from (2.2) that the eigenvalues of $(c-\rho(\Delta))^{d}$ are $\left(c+\pi\left(m+\frac{d}{2}\right)\right)^{d}$, $m=0,1, \cdots$, each of multiplicity $d_{m}:=\operatorname{dim}\left\{w^{\alpha},|\alpha|=m\right\}=\left(\begin{array}{c}d+m-1 \\ d-1\end{array}\right) \approx m^{d-1}$. The partial sums thus satisfy

$$
\sum_{m \leq N}\left(c+\pi\left(m+\frac{d}{2}\right)\right)^{-d} d_{m} \approx \frac{1}{(d-1) !} \sum_{m \leq N}\left(c+\pi\left(m+\frac{d}{2}\right)\right)^{-d} m^{d-1} \approx \frac{1}{(d-1) !} \log N,
$$

completing the proof.

Proposition 3.2. Let $F \in \mathcal{P} \mathcal{T}(-2 d, 1)$. Then the Weyl transform $\rho(F)$ is in the Macaev class $\mathcal{L}^{1, \infty}$.

Proof. By (3.5.6) in [10,

$$
\hat{\Delta}=-\frac{\pi^{2}}{4}|w|^{2},
$$

so $-\Delta \in \mathcal{P} \mathcal{T}(2,1)$, whence by (2.4) $(-\Delta)^{* d} \in \mathcal{P} \mathcal{T}(2 d, 1)$ and $(-\Delta)^{* d} * F \in \mathcal{P} \mathcal{T}(0,1)$. By the Calderón-Vaillancourt theorem [10, Theorem 3.1.3], the corresponding Weyl transform, $\rho(-\Delta)^{d} \rho(F)$, is bounded. Hence by the previous lemma, $\rho(F) \in \mathcal{L}^{1, \infty}$, since the Macaev class $\mathcal{L}^{1, \infty}$ is an ideal.

\section{Dixmier trace formula for Toeplitz operators}

Theorem 4.1. Let $F \in \mathcal{P} \mathcal{T}_{\text {rad }}(-2 d, 1)$ with the principal symbol $\sigma_{-2 d}(\hat{F})$ as defined in (2.3). Then the Dixmier trace $\operatorname{tr}_{\omega} \rho(F)$ is independent of $\omega$ and is given by

$$
\operatorname{tr}_{\omega} \rho(F)=\frac{\pi^{d}}{4^{d} d !} \int_{S} \hat{\sigma}_{-2 d}(F)(w),
$$

where $\int_{S}$ is the normalized integral over the unit sphere. 
Proof. The proof is quite similar to that of Connes [7] for pseudo-differential operators on compact manifolds. Namely, by [10, Theorem 4.2.5] and the defini-

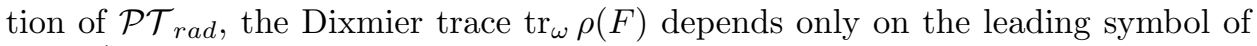
$\sigma_{-2 d}(\hat{F})$ and defines a positive measure on the unit sphere $S$ in $\mathbb{C}^{d}$. By the unitary invariance of $\rho(F)$ the measure has to be a constant multiple of the area measure. To find the constant we note that the symbol of $c \delta_{0}-\Delta, c>0$, is absolutely elliptic in the sense of (4.2.20) in [10, and thus by pp. 246-247 in [10] we can construct $F_{0} \in \mathcal{P} \mathcal{T}_{\text {rad }}(-2 d, 1)$ such that $\rho\left(F_{0}\right)=(c-\rho(\Delta))^{-d}$. The eigenvalue of $\rho\left(F_{0}\right)$ on the space of all $m$-homogeneous polynomials is, by the proof of Lemma 3.1,

$$
\frac{1}{\left(c+\pi\left(m+\frac{d}{2}\right)\right)^{d}} \text {. }
$$

Its Dixmier trace exists and is (noticing that the dimension of the space of homogeneous polynomials of degree $m \leq N$ is $\approx N^{d}$ )

$$
\operatorname{tr}_{\omega} \rho\left(F_{0}\right)=\frac{1}{\pi^{d} d !} .
$$

On the other hand, the principal symbol $\sigma_{-2 d}\left(F_{0}\right)$ is the constant function $\left(4 / \pi^{2}\right)^{d}|w|^{-2 d}$ by the definition (cf. (3.1)), whose integration over the sphere is $\left(4 / \pi^{2}\right)^{d}$. This completes the proof.

To apply our result to Toeplitz operators we need to introduce some more notation. We let

$$
\partial_{j}^{b}=\partial_{j}-\bar{z}_{j} R, \quad \bar{\partial}_{j}^{b}=\bar{\partial}_{j}-z_{j} \bar{R}
$$

be the boundary Cauchy-Riemann operators [17, where $R=\sum_{j=1}^{d} z_{j} \partial_{j}$ is the holomorphic radial derivative. As vector fields they are linearly dependent, to wit,

$$
\sum_{j=1}^{d} z_{j} \partial_{j}^{b}=0, \quad \sum_{j=1}^{d} \bar{z}_{j} \bar{\partial}_{j}^{b}=0 .
$$

Definition 4.2. We define a bracket $\{f, g\}_{b}$ for smooth functions $f$ and $g$ on $S$ by

$$
\{f, g\}_{b}:=\sum_{j=1}^{d}\left(\partial_{j}^{b} f \bar{\partial}_{j}^{b} g-\bar{\partial}_{j}^{b} f \partial_{j}^{b} g\right)
$$

and call it the boundary Poisson bracket.

Lemma 4.3. Let $F$ and $G$ be two functions in $\mathcal{P} \mathcal{T}_{\text {rad }}(0, \mu)$ with principal symbols

$$
\sigma_{0}(F)(z)=f\left(\frac{z}{|z|}\right), \quad \sigma_{0}(G)(z)=g\left(\frac{z}{|z|}\right)
$$

for $f$ and $g$ in $C^{\infty}(S)$. Then the principal symbol of $F * G-G * F$ is given by

$$
\sigma_{-2}(F * G-G * F)(z)=\frac{4}{\pi}\{f, g\}_{b}\left(\frac{z}{|z|}\right)|z|^{-2} .
$$


Proof. By the general result for the symbol calculus for pseudo-Toeplitz operators (cf. (2.2.5) in [10]), we have $F * G-G * F \in \mathcal{P} \mathcal{T}_{\text {rad }}(-2 \mu, \mu)$ with the principal symbol

$$
\left.\sigma_{-2}(F * G-G * F)(z)=\frac{4}{\pi}\left\{\sigma_{0}(F)\right), \sigma_{0}(G)\right\}(z),
$$

where $\{\cdot, \cdot\}$ is the ordinary Poisson bracket in complex coordinates

$$
\{\Psi, \Phi\}:=\sum_{j=1}^{d}\left(\partial_{j} \Psi \bar{\partial}_{j} \Phi-\partial_{j} \Phi \bar{\partial}_{j} \Psi\right) .
$$

The function $\sigma_{-2}(F * G-G * F)(z)$ is positive homogeneous of degree -2 . We need only to compute it for $z \in S$. Defining the Reeb vector field $E$ and the outward normal vector field $N$ in terms of the radial derivative $R$,

$$
E:=\frac{1}{2}(\bar{R}-R), \quad N:=\bar{R}+R,
$$

we can write

$$
R=-E+\frac{N}{2} .
$$

Note that $E$ is well-defined on $S$. The vector field $\partial_{j}^{b}-\bar{z}_{j} E$ is thus a well-defined vector field on $S$, and for any function $\Phi(z)=\phi\left(\frac{z}{|z|}\right)$ we have

$$
\partial_{j} \Phi(z)=\left(\partial_{j}^{b}+\bar{z}_{j} R\right) \Phi(z)=\left(\partial_{j}^{b}-\bar{z}_{j} E+\frac{\bar{z}_{j}}{2} N\right) \Phi(z)=\left(\partial_{j}^{b}-\bar{z}_{j} E\right) \phi(z),
$$

since $N \Phi(z)=0$ by homogeneity. Similarly $\bar{\partial}_{j} \Phi=\left(\partial_{j}^{b}+z_{j} E\right) \phi$ on $S$. From this it follows that for $z \in S$,

$$
\begin{aligned}
\left\{\sigma_{0}(F), \sigma_{0}(G)\right\}(z) & =\sum_{j=1}^{d}\left(\left(\partial_{j}^{b} f(z)-\bar{z}_{j} E f(z)\right)\left(\bar{\partial}_{j}^{b} g(z)+z_{j} E g(z)\right)\right. \\
& \left.\quad-\left(\bar{\partial}_{j}^{b} f(z)-\bar{z}_{j} E f(z)\right)\left(\partial_{j}^{b} g(z)+z_{j} E g(z)\right)\right) \\
& =\{f, g\}_{b},
\end{aligned}
$$

by using (4.1).

Theorem 4.4. Let $f_{1}, g_{1}, \cdots, f_{d}, g_{d}$ be smooth functions on $S, \tilde{f}_{1}, \tilde{g}_{1}, \cdots, \tilde{f}_{d}, \tilde{g}_{d}$ their smooth extensions to $B$ and $T_{\tilde{f}_{1}}, T_{\tilde{g}_{1}}, \cdots, T_{\tilde{f}_{d}}, T_{\tilde{g}_{d}}$ the associated Toeplitz operators on $\mathcal{H}_{\nu}$ for $\nu \geq d$. Then the product $\prod_{j=1}^{d}\left[T_{\tilde{f}_{j}}, T_{\tilde{g}_{j}}\right]$ is in the Macaev class and its Dixmier trace is given by

$$
\operatorname{tr}_{\omega} \prod_{j=1}^{d}\left[T_{\tilde{f}_{j}}, T_{\tilde{g}_{j}}\right]=\frac{1}{d !} \int_{S} \prod_{j=1}^{d}\left\{f_{j}, g_{j}\right\}_{b} .
$$

Proof. The proof is straightforward from the preceding lemma, formula (2.2.5) in [10] and Theorem 4.1.

We apply our result to Hankel operators with anti-holomorphic symbols. Let $f$ be a holomorphic function in a neighborhood of $B$ and $H_{\bar{f}} g=(I-P) \bar{f} g, g \in \mathcal{H}_{\nu}$ the Hankel operator. Then

$$
\left[T_{\bar{f}}, T_{f}\right]=\left[T_{f}^{*}, T_{f}\right]=\left|H_{\bar{f}}\right|^{2}=H_{\bar{f}}^{*} H_{\bar{f}} .
$$


Corollary 4.5. Let $f$ be as above. Then the Hankel operator is in $\mathcal{L}^{2 d, \infty}$; equivalently the commutator $\left[T_{\bar{f}}, T_{f}\right]$ is in $\mathcal{L}^{d, \infty}$ and we have

$$
\operatorname{tr}_{\omega}\left|H_{\bar{f}}\right|^{2 d}=\operatorname{tr}_{\omega}\left(\left[T_{\bar{f}}, T_{f}\right]^{d}\right)=\frac{1}{d !} \int_{S}\left(|\nabla f|^{2}-|R f|^{2}\right)^{d} .
$$
is

Notice that $H_{\bar{f}}$ is in the Schatten class $\mathcal{L}^{p}$ for $p>2 d$ and that its Schatten norm

$$
\left\|H_{\bar{f}}\right\|_{p}^{p} \approx \int_{B}\left(1-|z|^{2}\right)^{p}\left(|\nabla f|^{2}-|R f|^{2}\right)^{\frac{p}{2}} d m(z)
$$

see [3] and 19] for the Bergman space case $(\nu=d+1)$ and the Hardy space case $(\nu=d)$. Our resulting formula thus provides a limiting result of the above estimates, and it is interesting to note that the estimate has an equality as its limit for $p \rightarrow 2 d$.

\section{REFERENCES}

[1] J. Arazy, S. D. Fisher, and J. Peetre, Hankel operators on weighted Bergman spaces, Amer. J. Math. 110 (1988), no. 6, 989-1053. MR970119 (90a:47067)

[2] J. Arazy, S. D. Fisher, S. Janson, and J. Peetre, An identity for reproducing kernels in a planar domain and Hilbert-Schmidt Hankel operators, J. Reine Angew. Math. 406 (1990), 179-199. MR1048240 (91b:47050)

[3] - Membership of Hankel operators on the ball in unitary ideals, J. London Math. Soc. (2) 43 (1991), no. 3, 485-508. MR.1113389 (93c:47030)

[4] P. Boggiatto and F. Nicola, Non-commutative residues for anisotropic pseudo-differential operators in $\mathbb{R}^{n}$, J. Funct. Anal. 203 (2003), no. 2, 305-320. MR.2003350 (2004g:58036)

[5] M. Feldman and R. Rochberg, Singular value estimates for commutators and Hankel operators on the unit ball and the Heisenberg group, Analysis and partial differential equations, Lecture Notes in Pure and Appl. Math., vol. 122, Dekker, New York, 1990, pp. 121-159. MR:1044786(91e:47024)

[6] V. Guillemin, Toeplitz operators in $n$ dimensions, Integral Equations Operator Theory 7 (1984), no. 2, 145-205. MR750217 (86i:58130)

[7] A. Connes, The action functional in noncommutative geometry, Comm. Math. Phys. 117 (1988), no. 4, 673-683. MR.953826 (91b:58246)

[8] A. Connes, Noncommutative geometry, Academic Press Inc., San Diego, CA, 1994. MR.1303779 (95j:46063)

[9] J. W. Helton and R. E. Howe, Traces of commutators of integral operators, Acta Math. 135 (1975), no. 3-4, 271-305. MR0438188 (55:11106)

[10] R. Howe, Quantum mechanics and partial differential equations, J. Funct. Anal. 38 (1980), no. 2, 188-254. MR.587908 (83b:35166)

[11] S. Janson, J. Peetre, and R. Rochberg, Hankel forms and the Fock space, Rev. Mat. Iberoamericana 3 (1987), no. 1, 61-138. MR1008445 (91a:47029)

[12] S. Janson, H. Upmeier, and R. Wallstén, Schatten-norm identities for Hankel operators, J. Funct. Anal. 119 (1994), no. 1, 210-216. MR1255278 (95a:47021)

[13] J. Peetre, On the $S_{4}$-norm of a Hankel form, Rev. Mat. Iberoamericana 8 (1992), no. 1, 121-130. MR1178451 (93f:47027)

[14] V. V. Peller, Hankel operators and their applications, Springer Monographs in Mathematics, Springer-Verlag, New York, 2003. MR.1949210 (2004e:47040)

[15] R. Ponge, Noncommutative residue invariants for $C R$ and contact manifolds, J. Reine Angew. Math. 614 (2008), 117-151. MR2376284 (2008j:58039)

[16] R. Rochberg, Trace ideal criteria for Hankel operators and commutators, Indiana Univ. Math. J. 31 (1982), no. 6, 913-925. MR674875 (84d:47036)

[17] W. Rudin, Function theory in the unit ball of $\mathbb{C}^{n}$, Springer-Verlag, New York-Berlin, 1980. MR601594 (82i:32002) 
[18] E. M. Stein, Harmonic analysis: Real-variable methods, orthogonality, and oscillatory integrals, Princeton Mathematical Series, vol. 43, Princeton University Press, Princeton, NJ, 1993, with the assistance of Timothy S. Murphy, Monographs in Harmonic Analysis, III. MR.1232192 (95c:42002)

[19] K. H. Zhu, Schatten class Hankel operators on the Bergman space of the unit ball, Amer. J. Math. 113 (1991), no. 1, 147-167. MR1087805 (91m:47036)

Mathematics Institute AS ČR, Žitná 25, 11567 Prague 1, Czech Republic - and Mathematics Institute, Silesian University, Na Rybníč́u 1, 74601 Opava, Czech Republic E-mail address: englis@math.cas.cz

Department of Mathematics, Fudan University, Shanghai 200433, People's Republic OF CHINA

E-mail address: kyguo@fudan.edu.cn

Department of Mathematics, Chalmers University of Technology and Göteborg University, Göteborg, Sweden

E-mail address: genkai@chalmers.se 